ARTICLE

httpst//doi.org/10.1038/s41467-019-11895-2

\title{
Caspase-11 signaling enhances graft-versus-host disease
}

\author{
Yanyan Lu ${ }^{1}$ Ran Meng ${ }^{1}$, Xiangyu Wang ${ }^{1}$, Yajing $\mathrm{Xu}^{2}$, Yiting Tang ${ }^{3}$, Jianfeng Wu${ }^{4}$, Qianqian Xue ${ }^{1}$ Songlin Yu ${ }^{1}$, \\ Mingwu Duan ${ }^{1}$, Dongyong Shan ${ }^{1}$, Qingde Wang ${ }^{5}$, Haichao Wang (1) ${ }^{6}$, Timothy R. Billiar ${ }^{5}$, Xianzhong Xiao ${ }^{7,8}$, \\ Fangping Chen ${ }^{1} \&$ Ben Lu (iD) ${ }^{1,7,8}$
}

Acute graft-versus-host disease (GVHD) remains a major obstacle for the wider usage of allogeneic hematopoietic stem cell transplantation (allo-HSCT), which is an effective therapy for hematopoietic malignancy. Here we show that caspase-11, the cytosolic receptor for bacterial endotoxin (lipopolysaccharide: LPS), enhances GVHD severity. Allo-HSCT markedly increases the LPS-caspase-11 interaction, leading to the cleavage of gasdermin D (GSDMD). Caspase-11 and GSDMD mediate the release of interleukin-1 $\alpha$ (IL-1 $\alpha$ ) in allo-HSCT. Deletion of Caspase-11 or Gsdmd, inhibition of LPS-caspase-11 interaction, or neutralizing IL-1 $\alpha$ uniformly reduces intestinal inflammation, tissue damage, donor T cell expansion and mortality in allo-HSCT. Importantly, Caspase-11 deficiency does not decrease the graft-versus-leukemia (GVL) activity, which is essential to prevent cancer relapse. These findings have major implications for allo-HSCT, as pharmacological interference with the caspase-11 signaling might reduce GVHD while preserving GVL activity.

\footnotetext{
${ }^{1}$ Department of Hematology and Critical Care Medicine, The 3rd Xiangya Hospital, Central South University, Changsha 410000, P.R. China. ${ }^{2}$ Department of Hematology, Xiangya Hospital, Central South University, Changsha 410008, P.R. China. ${ }^{3}$ Department of Physiology, School of Basic Medical Science, Central South University, Changsha, Hunan Province 410000, P.R. China. ${ }^{4}$ State Key Laboratory of Cellular Stress Biology Innovation Center for Cell Signaling Network School of Life Sciences, Xiamen University, Xiamen, Fujian Province 361005, P.R. China. ${ }^{5}$ Department of Surgery, University of Pittsburgh Medical Center, Pittsburgh, PA 15213, USA. ${ }^{6}$ The Feinstein Institute for Medical Research, Northwell Health, 350 Community Drive, Manhasset, NY 11030 , USA.

${ }^{7}$ Key Laboratory of Sepsis Translational Medicine of Hunan, Central South University, Changsha, Hunan Province 410000, P.R. China. ${ }^{8}$ Department of Pathophysiology, School of Basic Medical Science, Central South University, Changsha, Hunan Province 410000, P.R. China. Correspondence and requests for materials should be addressed to B.L. (email: xybenlu@csu.edu.cn)
} 
T he success of allogeneic hematopoietic stem cell transplantation (allo-HSCT), a standard therapy for conditions such as hematopoietic malignancies and inherited hematopoietic disorders, is limited by the mortality and morbility associated with graft-versus-host disease $(\mathrm{GVHD})^{1,2}$. In alloHSCT, irradiation- or chemotherapy-induced gastrointestinal (GI) damage allows the translocation of bacteria or lipopolysaccharide (LPS), the major cell-wall component of Gramnegative bacteria, from GI tract into the systemic circulation ${ }^{1,3}$. Serum and tissue levels of LPS are closely correlated with GVHD severity and mortality 3,4 . LPS antagonism reduces GVHD while preserving graft-versus-leukemia (GVL) activity in experimental models ${ }^{5}$. Further, antibodies against LPS or Gram-negative bacteria significantly reduces the incidence of GVHD in clinical trials $^{6,7}$. It has been proposed that LPS or Gram-negative bacteria enhance GVHD through toll-like receptor 4 (TLR4), a wellestablished cell surface LPS receptor. However, allo-HSCT recipient mice that express a signaling-deficient Tlr4 mutant developed fulminant GVHD and increased intestinal damage compared to their wild-type (WT) counterparts ${ }^{8}$. These observations raise a possibility that LPS or Gram-negative bacteria might enhance GVHD through TLR4-independent signaling pathways.

Caspase-11 is a cytosolic LPS receptor that senses various Gram-negative bacteria infections ${ }^{9-15}$. Upon activation by intracellular LPS, caspase-11 oligomerizes into protein complexes and enzymatically cleaves gasdermin D (GSDMD) into pore-forming peptides, leading to a lytic form of cell death, termed pyroptosis $^{16-18}$. This process destroys the intracellular niche for microbes and triggers inflammation by releasing alarmins, such as interleukin-1a (IL-1 1$)^{12,13,15}$. Loss of caspase-11 renders mice susceptible to Burkholderia pseudomallei, a Gram-negative bacterium endemic to Southeast Asia that causes melioidosis ${ }^{12}$. However, over-activation of caspase-11 in endotoxemia or polymicrobial sepsis leads to organ injury and lethality ${ }^{10,19-22}$. Dysregulated activation of caspase-11 also contributes to the pathogenesis of age-related macular degeneration ${ }^{23}$. Based on these findings and the demonstrated role of LPS in the development of GVHD, we hypothesize that caspase-11 signaling contributes to the pathogenesis of GVHD in allo-HSCT.

In this study, we show that the LPS-caspase-11 interaction is important for the development of GVHD in allo-HSCT. Recognition of LPS by caspase-11 leads to the cleavage of GSDMD, which is essential for the release of IL-1 $\alpha$ after bone marrow transplantation (BM). Disruption of the LPS-caspase-11 interaction, deletion of Caspase-11 or Gsdmd, or neutralization of IL$1 \alpha$ uniformly reduces intestinal inflammation, donor $\mathrm{T}$ cell expansion, and mortality in allo-HSCT. Moreover, Caspase-11 deficiency does not compromise the GVL effect. These findings suggest that pharmacological inhibition of the caspase-11 signaling might reduce GVHD while preserving GVL activity in allo-HSCT.

\section{Results}

Caspase-11 enhances GVHD in allo-HSCT. To determine the role of caspase-11 in GVHD following allo-HSCT, T cells purified from WT mice (on a BALB/c background, H2d) were transplanted together with BM into lethally irradiated major histocompatibility complex (MHC)-mismatched WT or Caspase-11deficient recipient mice (on a $\mathrm{B} 6$ background, $\mathrm{H} 2 \mathrm{~b}$, provided by the University of Pittsburgh). We observed that genetic deletion of Caspase-11 in recipients significantly improved survival (Fig. 1a). These findings were reproduced when we used a different strain of Caspase-11-deficient mice (on a B6 background, from the Jackson laboratory) or their WT littermates (Fig. 1b).
Furthermore, similar observations were made when mice were pre-conditioned with busulfan and cyclophosphamide (BU/CY) chemotherapy (Fig. 1c). Caspase-11-deficient recipients had markedly fewer pathological injuries (in liver, lung, small intestine, and colon) (Fig. 1d), and displayed significantly lower GVHD pathological scores compared with WT controls (Fig. 1e and Supplementary Fig. 1a). However, pre-transplant radiation resulted in similar weight loss and tissue injury in Caspase-11deficient recipients and their WT controls (Supplementary Fig. $1 b, c)$. We next examined whether caspase-11 in donor cells contributes to the pathogenesis of GVHD. Purified T cells and BM from Caspase-11-deficient mice or their WT littermates (on a B6 background, $\mathrm{H} 2 \mathrm{~b}$ ) were transplanted into lethally irradiated recipient mice on a BALB/c background (H2d). Deletion of Caspase-11 in donor cells resulted in a delayed mortality and slightly improved GVHD pathological scores in WT recipients (Fig. If and Supplementary Fig. 1d, e). We established that caspase-11 expressed in the non-hematopoietic compartment plays the dominant role in promoting GVHD lethality in alloHSCT, while caspase-11 expressed in the hematopoietic compartment plays a minor role (Supplementary Fig. 1f). Caspase-11 deficiency in both recipients and donor BM cells further decreased allogeneic $\mathrm{T}$ cell-mediated lethality (Supplementary Fig. 1f).

Caspase-11 enhances donor $T$ cell expansion. The tissue injury associated with GVHD is caused by the activation of allogeneic donor $\mathrm{T}$ cells. To test whether caspase-11 enhances allogeneic donor T cell expansion following HSCT, we used BALB/c donor $\mathrm{T}$ cells that express luciferase linked to the $\beta$-actin promoter. Two weeks after allo-HSCT, Caspase-11-deficient or WT recipients were subjected to bioluminescence (bioluminescent imaging (BLI)) to measure donor $\mathrm{T}$ cell expansion. Quantification of whole-body or intestinal BLI intensity indicated that Caspase-11deficient recipients had significantly reduced $\mathrm{T}$ cell expansion, as compared to that of WT recipients (Fig. 2a, b). Activation of allogeneic $\mathrm{T}$ cells in GVHD is associated with increased production of interferon- $\gamma$ (IFN- $\gamma$ ) and other proinflammatory cytokines, such as IL-17. Neutrophil recruitment into the tissues promotes allogeneic donor $\mathrm{T}$ cell expansion and exacerbates GVHD-related tissue injury and mortality ${ }^{1}$. In line with these findings, serum levels of IFN- $\gamma$ and neutrophil infiltration in the colon of Caspase-11-deficient recipients were significantly lower than those in WT recipients (Fig. 2c, d). Caspase-11 deficiency in recipient mice was also associated with significantly lower serum levels of IL-17 and IL-6 (Supplementary Fig. 2a-c) following alloHSCT. Deletion of Caspase-11 significantly inhibited CD4 T cell T-helper type 1 (Th1) and Th17 polarization in the intestine (Supplementary Fig 2d, e). Taken together, our data demonstrate that Caspase-11 promotes neutrophil infiltration, intestinal inflammation, tissue damage, donor $\mathrm{T}$ cell expansion and mortality in allo-HSCT.

LPS-caspase-11 interaction enhances GVHD. Caspase-11 is a cytosolic LPS receptor that is constitutively expressed in intestinal tissues $^{24}$. To assess the LPS-caspase-11 interaction in allo-HSCT, we performed the proximity-ligation assay (PLA) that visualizes the physical interaction between two molecules ${ }^{22}$. Allo-HSCT markedly increased the LPS-caspase-11 interaction in the intestine of WT mice (Fig. 3a). As expected, the interaction was lost with genetic deletion of Caspase-11 (Fig. 3a). LPS-caspase-11 complexes predominantly co-localized with an epithelial marker (Supplementary Fig. 3a), further supporting the notion that caspase-11 expressed in the non-hematopoietic compartment plays the dominant role in promoting GVHD following 
a

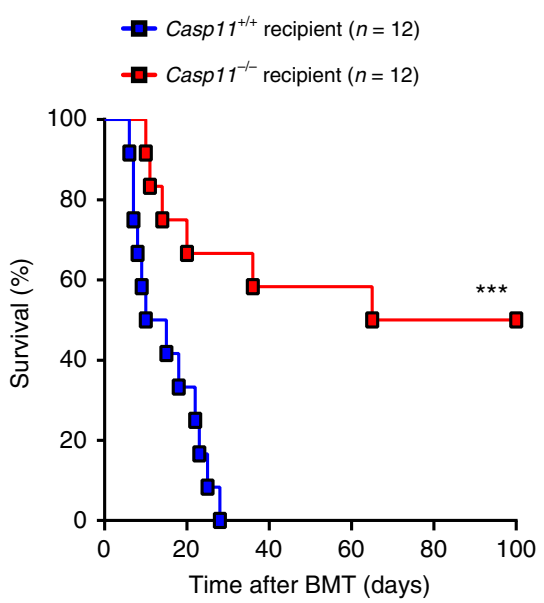

C

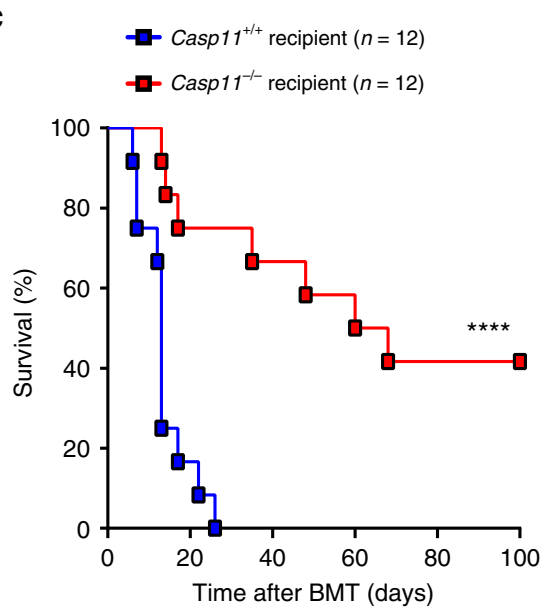

$$
\begin{aligned}
& \text { - Casp } 11^{+/+} \text {recipient } \\
& \text { O Casp } 11^{-/-} \text {recipient }
\end{aligned}
$$

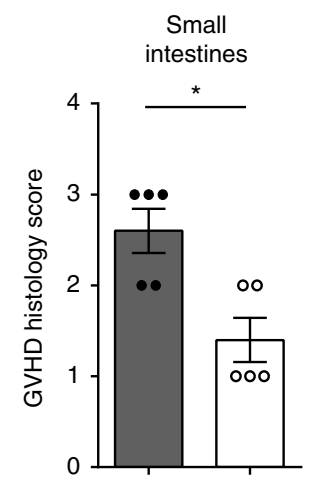

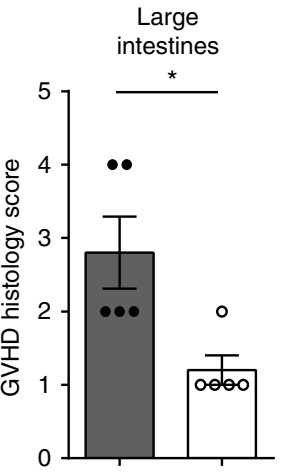

b
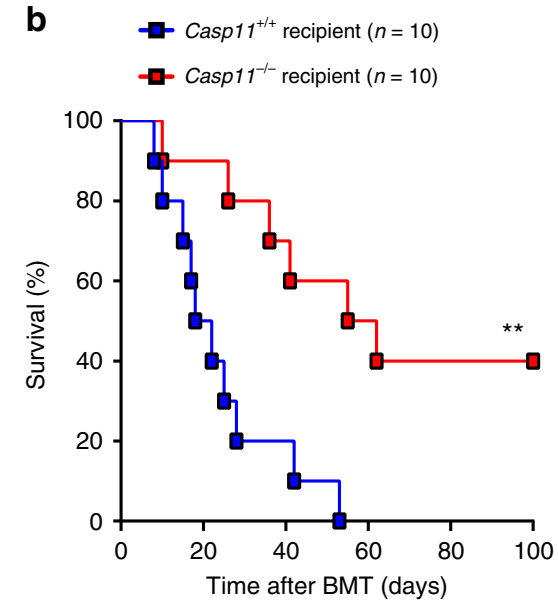

d
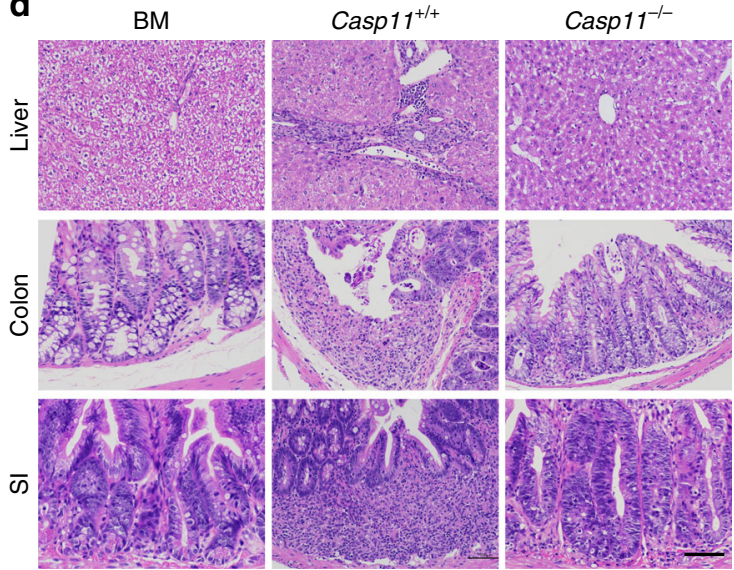

f
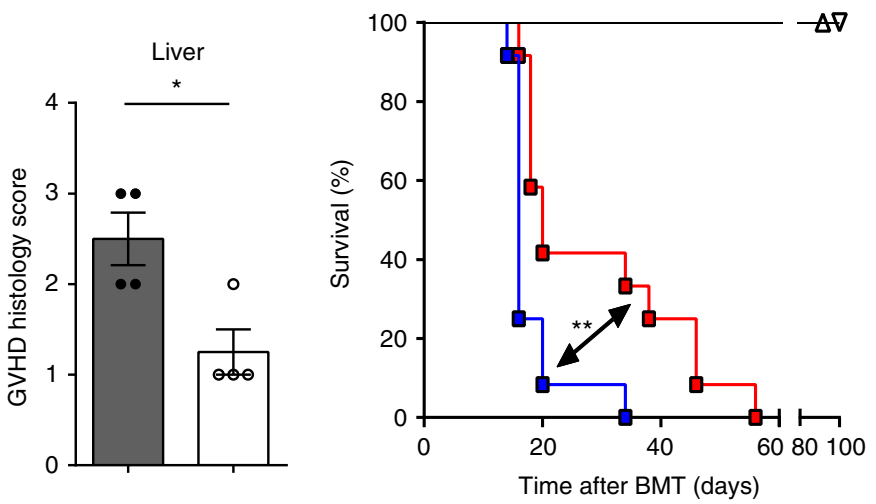

allo-HSCT. Recognition of LPS by caspase-11 requires guanylatebinding proteins $(\mathrm{GBPs})^{13,25}$. In line with these findings, LPS-caspase-11 interaction in the intestinal epithelium was not observed when GBPchr3 KO (knockout) mice ${ }^{13}$, which lack GBP1, 2, 3, 5, and 7, were used as HSCT recipients (Fig. 3b). We also observed that deletion of GBPs in recipients significantly improved survival (Fig. 3c), and markedly reduced serum levels of IFN- $\gamma$ as well as neutrophil infiltration into the colon (Supplementary Fig. 3b, c). Accordingly, GBPchr3 KO recipients displayed significantly improved GVHD pathological scores as

compared to WT recipients (Fig. 3d and Supplementary Fig. 3d). GBPchr3 KO recipients also exhibited reduced $\mathrm{T}$ cell expansion to a level similar to that seen in Caspase-11-deficient recipients (Fig. 3e, f).

To further confirm that the interaction of LPS with caspase-11 promotes GVHD, mice were treated with LPS from the photosynthetic bacterium Rhodobacter sphaeroides (LPS-RS), the penta-acylated LPS that competitively inhibits the LPS-caspase-11 interaction ${ }^{22}$ (Supplementary Fig. 3a). Treatment of LPS-RS significantly promoted survival in WT but not 
Fig. 1 Caspase-11 (Casp11) enhances graft-versus-host disease (GVHD) in allogeneic hematopoietic stem cell transplantation (allo-HSCT). a Percentage survival of Casp11 $1 /+$ recipient mice versus Casp11 $/$ - recipient mice (BALB/c $\rightarrow$ C57BL/6 combination) receiving BALB/c bone marrow (BM) and T cells is shown ( $n=12$ in each group). ( ${ }^{\star \star \star} P=0.0008$; differences in animal survival were analyzed by log-rank test). b Survival of Casp11+/+ and Casp11-/- (Jax) recipients receiving allo-HSCT from BALB/c donors after TBI (total body irradiation) based conditioning is shown ( $n=10$ in each group) ( $* \star P=0.0022$; differences in animal survival were analyzed by log-rank test). c Survival of Casp11 ${ }^{+/+}$recipient mice versus Casp11-/- recipient mice receiving allo-HSCT from BALB/c donors after chemotherapy (busulfan/cyclophosphamide (BU/CY))-based conditioning is shown ( $n=12$ in each group). The experiment was performed twice and data were pooled. ( ${ }^{\star \star \star *} P<0.0001$, differences in animal survival were analyzed by log-rank test). $\mathbf{d}$ Photomicrographs depicting the average disease score morphology from one representative experiment out of two separate experiments are depicted. Scale bar: $100 \mu \mathrm{m}$. BM represents C57BL/6 recipients receiving BALB/c BM group. Casp11+/+ and Casp11-/- recipients represent mice receiving BALB/c BM and T cells group. e Histological analysis of the small intestines, large intestines, and liver from Casp11+/+ and Casp11-/- recipient mice receiving BALB/c BM and T cells for GVHD severity on day 14. Data are presented as mean \pm SEM. ${ }^{\star} P<0.05$. An unpaired Student's $t$ test (two-sided) was used. $\mathbf{f}$ Survival of wild-type (WT) recipient mice $(\mathrm{C} 57 \mathrm{BL} / 6 \rightarrow \mathrm{BALB} / \mathrm{c}$ combination) receiving Casp11 //+ versus Casp11-/- BM with or without $\mathrm{T}$ cells is shown. The experiment was performed twice and the results were pooled. Casp11+/+ versus Casp11 ${ }^{-/-}\left({ }^{\star} P=0.0054\right.$; differences in animal survival were analyzed by log-rank test $)$
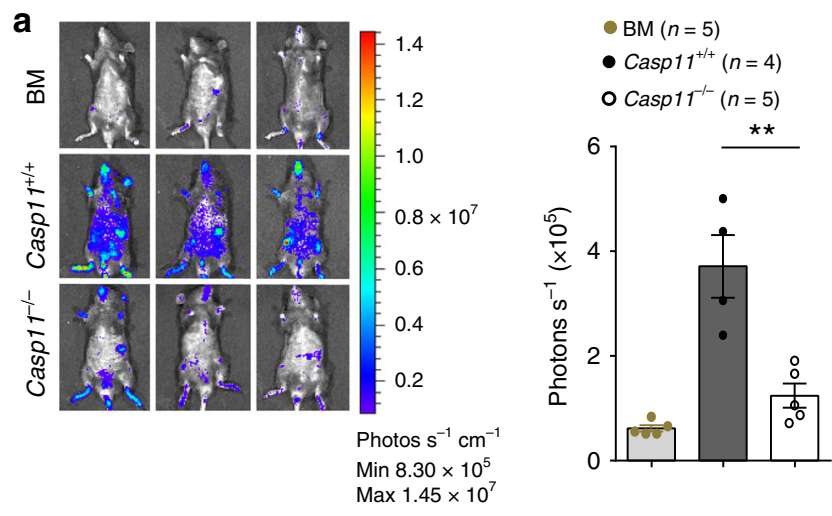

- Casp $11^{+/+}$recipient $(n=5)$
O Casp $11^{-/-}$recipient $(n=5)$

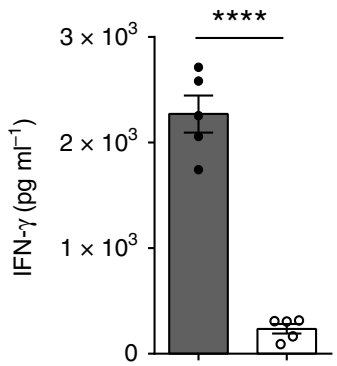

b

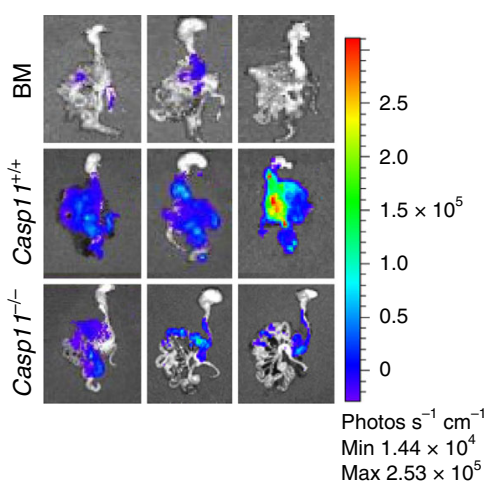

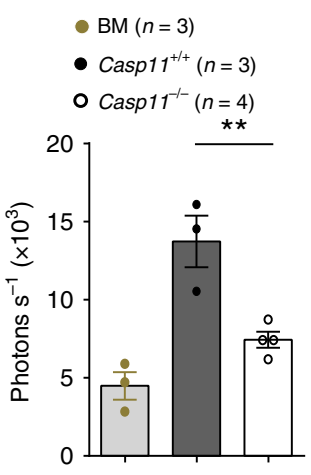

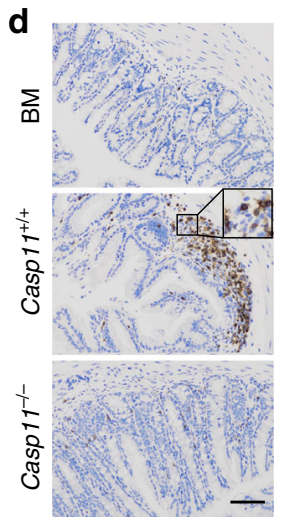

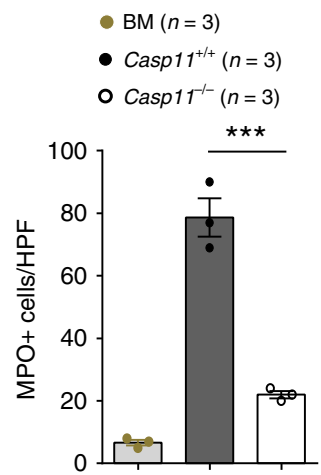

Fig. 2 Caspase-11 enhances donor T cell expansion in allogeneic hematopoietic stem cell transplantation (allo-HSCT). a Fourteen days post transplant, Casp11 $1 /+$ and Casp11 1 - recipients receiving BALB/c bone marrow and T cells were subjected to intraperitoneal luciferin injection and whole-body bioluminescent imaging (BLI) are depicted. Region of interest gating and signal normalization with Living Image software. Average representative wholebody images, and average bioluminescence intensities \pm SEM were depicted. ( ${ }^{\star \star} P=0.0040$; an unpaired Student's $t$ test (two-sided) was used). b Fourteen days post transplant, Casp11 $1 /+$ and Casp11-/- recipients receiving BALB/c bone marrow and T cells were subjected to intraperitoneal luciferin injection and whole-body BLI followed by a second luciferin injection, euthanization, organ removal, and BLI of graft-versus-host disease (GVHD) target organs of intestines. Bioluminescence was quantified using whole organ region of interest gating and signal normalization with Living Image software. Average representative ex vivo organ images, and average bioluminescence intensities \pm SEM were depicted. $\left({ }^{\star \star} P=0.0089\right.$; an unpaired Student's $t$ test (two-sided) was used). c Interferon- $\gamma$ (IFN- $\gamma$ ) in the serum of mice receiving BM and T cells (groups described in $\mathbf{a}, \mathrm{BALB} / \mathrm{C}$ (donor), C57BL/6 (recipient) model, day 5 after allo-HSCT). Data (mean \pm SEM) are pooled from two independent experiments ( ${ }^{\star \star \star \star} P<0.0001$, an unpaired Student's $t$ test (two-sided) was used). $\mathbf{d}$ Representative colon tissue section of Casp11+/+ recipient mice versus Casp11-/- recipient mice (BALB/c $\rightarrow$ C $57 \mathrm{BL} / 6$ combination) receiving $\mathrm{BALB} / \mathrm{c}$ bone marrow and T cells is shown. Staining in brown is for myeloperoxidase as indicated for the respective tissues. The frequency of myeloperoxidase positive (MPO+) cells per high power field (HPF) was significantly lower in the caspase-11-deficient recipients than those of in wild-type (WT) recipients. Data are presented as mean \pm SEM ( ${ }^{\star \star \star} P=0.0008$, an unpaired Student's $t$ test (two-sided) was used). Scale bar: $100 \mu \mathrm{m}$

GBPchr3 KO recipient mice following allo-HSCT (Fig. 3g). Conversely, intraperitoneal (i.p.) administration of LPS after alloHSCT significantly promoted GVHD lethality in WT but not in Caspase-11-deficient or GBPchr3 KO recipient mice (Fig. 3h). Oral administration of ciprofloxacin significantly decreased the circulating LPS levels (Supplementary Fig. 4a, b). Accordingly, ciprofloxacin treatment significantly improved survival in WT but not in Caspase-11-deficient recipient mice (Supplementary Fig. 4c). Taken together, these data indicate that LPS-caspase-11 interaction enhances GVHD in allo-HSCT. 
a
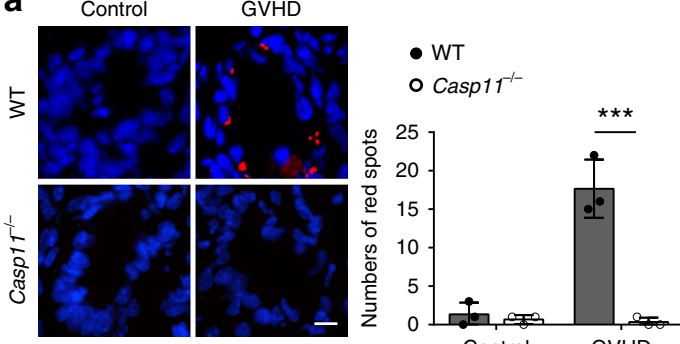

Control b

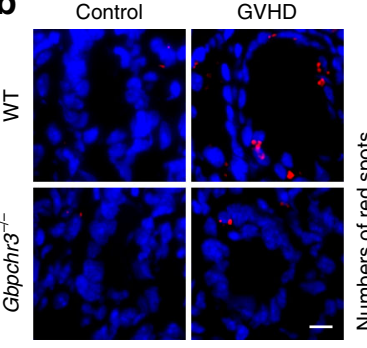

- WT

- $\mathrm{GBPChr}^{-1-}$

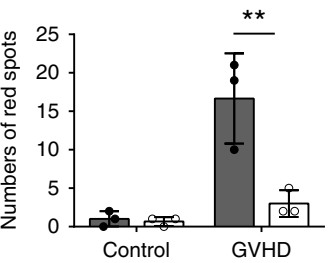

C

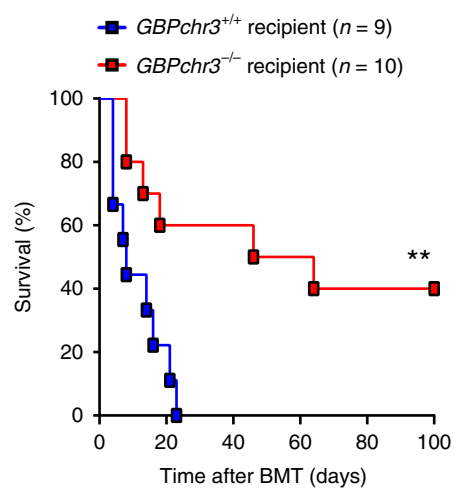

d

- $\mathrm{GBPChr}^{+/+}$recipient $(n=4)$

- $\mathrm{GBPChr}^{-/-}$recipient $(n=5)$

Small

intestines
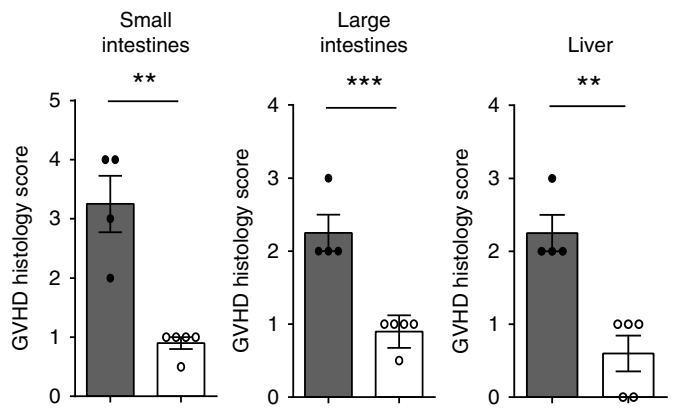

e

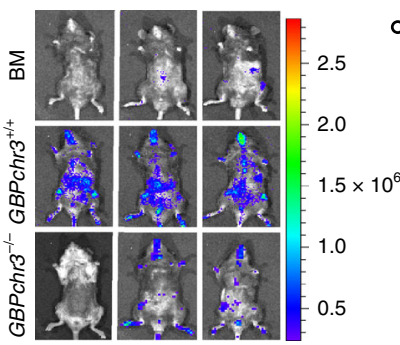

- $\mathrm{BM}(n=5)$

- $G B P c h r 3^{+/+}$recipient $(n=5)$

- $\mathrm{GBPChr}^{-/-}$recipient $(n=5)$

Photos $\mathrm{s}^{-1} \mathrm{~cm}^{-1}$

$\operatorname{Max} 2.87 \times 10^{6}$

g
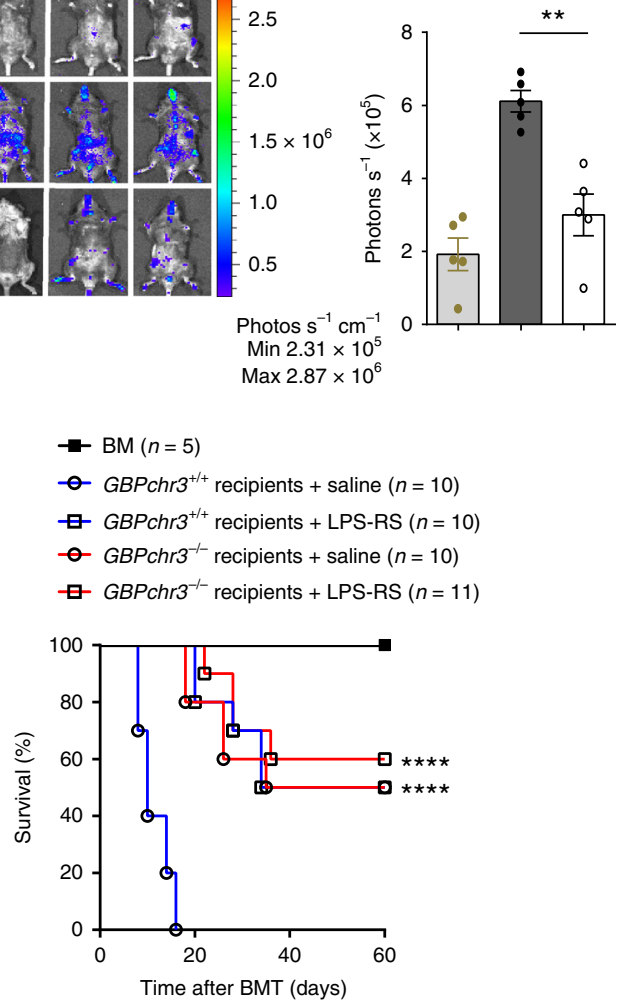

Caspase-11 enhances GVHD through GSDMD. GSDMD is a caspase-11 substrate that directly triggers pyroptosis ${ }^{16,17}$. AlloHSCT leads to the cleavage of GSDMD in the intestine in WT recipients. Deletion of Caspase-11 or GBPs in recipient mice markedly decreased GSDMD cleavage in allo-HSCT (Fig. 4a). Treatment of LPS-RS significantly inhibited and LPS administration enhanced GSDMD cleavage in the intestine after alloHSCT (Fig. 4a). Next, we determined whether caspase-11 f

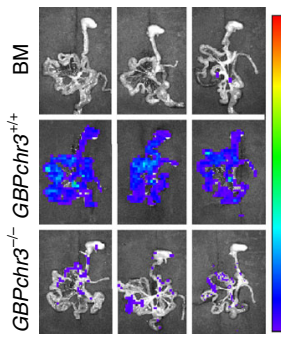

- $\operatorname{BM}(n=3)$

- $G B P c h r 3^{+/+}$recipient $(n=5)$

- $\mathrm{GBPChr}^{-1-}$ recipient $(n=4)$

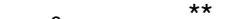

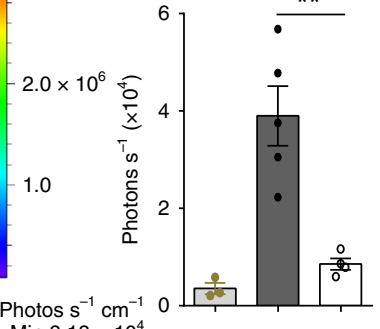
Min $8.18 \times 10^{4}$ $\operatorname{Max} 3.21 \times 10^{6}$

h

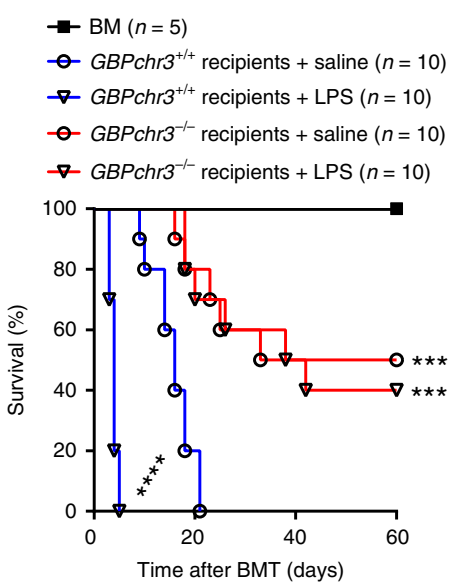

enhances GVHD through GSDMD in the MHC-mismatched allo-HSCT. Deletion of Gsdmd significantly improved survival (Fig. 4b), and markedly reduced serum IFN- $\gamma$ levels (Supplementary Fig. 5a) and intestinal neutrophil infiltration (Supplementary Fig. 5b). Gsdmd deficiency significantly improved GVHD pathological scores in a manner similar to Caspase-11 deficiency (Fig. 4c and Supplementary Fig. 5c) and was associated with reduced $\mathrm{T}$ cell expansion as using luciferase-expressing 
Fig. 3 Lipopolysaccharide (LPS)-caspase-11 interaction enhances graft-versus-host disease (GVHD) in allogeneic hematopoietic stem cell transplantation (allo-HSCT). a The physical interactions between caspase-11 and LPS were visualized as the red spots by proximity-ligation assay (PLA) in the intestinal

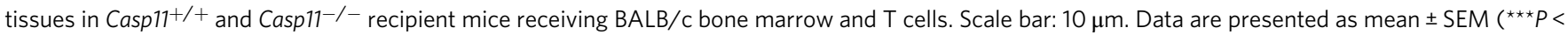
0.001 , an unpaired Student's $t$ test (two-sided) was used). $\mathbf{b}$ The physical interactions between caspase-11 and LPS were visualized as the red spots by PLA in the intestinal tissues in Gbpchr3 $3^{+/+}$and Gbpchr3 ${ }^{-/-}$recipient mice receiving BALB/c bone marrow and T cells. Scale bar: $10 \mu \mathrm{m}$. Data are presented as mean \pm SEM ( ${ }^{\star \star} P<0.01$, an unpaired Student's $t$ test (two-sided) was used). c Survival of C57BL/6 mice is shown (Gbpchr3 ${ }^{+/+}$versus Gbpchr3-/-, ${ }^{\star \star} P=$ 0.0045; differences in animal survival were analyzed by log-rank test). d Histopathological GVHD severity of C57BL/6 mice after receiving BALB/c bone marrow and T cells (BALB/c $\rightarrow$ C57BL/6) is shown (Gbpchr3 ${ }^{+/+}$versus Gbpchr3 ${ }^{-/-}$). Data are presented as mean $\left({ }^{\star \star} P<0.01 ;{ }^{\star \star \star} P<0.001 ;\right.$ an unpaired Student's $t$ test (two-sided) was used). e Fourteen days post transplant, Gbpchr3 ${ }^{+/+}$and Gbpchr3-/- recipients were subjected to intraperitoneal luciferin injection and whole-body bioluminescent imaging (BLI) are depicted. Average representative whole-body images and bioluminescence intensities \pm SEM are depicted ( ${ }^{\star \star} P=0.0013$; an unpaired Student's $t$ test (two-sided) was used). f Fourteen days post transplant, Gbpchr3 $3^{+/+}$and $\mathrm{Gbpchr} 3^{-/-}$recipients were subjected to intraperitoneal luciferin injection, whole-body BLI, and BLI of intestines. Average representative ex vivo organ images and average bioluminescence intensities \pm SEM are depicted ( ${ }^{\star \star} P=0.0034$; an unpaired Student's $t$ test (two-sided) was used). $\mathbf{g}$ Percentage survival of Gbpchr3 ${ }^{+} /+$ and Gbpchr3 ${ }^{-/-}$recipient mice receiving BALB/c bone marrow and T cells with treatment of lipopolysaccharide from the photosynthetic bacterium Rhodobacter sphaeroides (LPS-RS) or saline is shown. Differences in animal survival were analyzed by log-rank test. (Gbpchr3 $3^{+/+}+$saline versus Gbpch3 ${ }^{-/-}$ + saline, ${ }^{\star \star \star \star P}<0.0001 ;$ Gbpchr3 ${ }^{+/+}+$saline versus Gbpchr3 ${ }^{+/+}+$LPS-RS, ${ }^{\star \star \star \star P}<0.0001 ;$ Gbpchr3 ${ }^{+/+}+$saline versus Gbpchr3-/- + LPS-RS, $\star \star \star \star \mathrm{P}<0.0001)$. h Percentage survival of Gbpchr3 ${ }^{+/+}$and $\mathrm{Gbphr}^{-/-}$recipient mice with multiple injections of LPS or saline is shown. Differences in animal survival were analyzed by log-rank test. (Gbpchr3 ${ }^{+/+}+$saline versus Gbpchr3 ${ }^{-/-}+$saline, ${ }^{\star \star \star} P=0.0004 . ;$ Gbpchr3 ${ }^{+/+}+$saline versus Gbpchr3 $^{+/+}+$LPS, ${ }^{\star \star \star \star} P<0.0001 ;$ Gbpchr3 ${ }^{+/+}+$saline versus Gbpchr3 ${ }^{-/-}+$LPS, $\left.{ }^{\star \star \star} P=0.0002\right)$

T cells as described above (Fig. 4d). Treatment of LPS-RS significantly promoted survival in WT but not in Gsdmd KO recipient mice during allo-HSCT (Fig. 4e). Further, LPS treatment significantly promoted lethality in WT but not in Gsdmd KO recipient mice during allo-HSCT (Fig. 4f). These findings show that caspase-11 promotes GVHD through GSDMD in allo-HSCT.

Caspase-11 and GSDMD mediates IL-1 $\alpha$ release in GVHD. Caspase-11 activation and subsequent GSDMD cleavage lead to the release of IL-1 $\alpha$ in endotoxemia and bacterial sepsis ${ }^{10,16,22}$. We next determined whether caspase-11 and GSDMD mediates IL-1 $\alpha$ release in GVHD. Serum levels of IL-1 $\alpha$ were significantly increased in allo-HSCT (Fig. 5a-c). Deletion of Caspase-11 or Gsdmd in recipients markedly reduced IL-1 $\alpha$ release, indicating that caspase-11- and GSDMD-expressing non-hematopoietic cells (e.g., epithelial cells) are the major source of IL-1 1 after alloHSCT (Fig. 5a-c). Serum levels of IL-1 $\alpha$ were correlated with GVHD pathological scores in mouse models (Fig. 5d). Importantly, we prospectively analyzed the serum levels of 50 patients undergoing allo-HSCT (Supplementary Table 1) and found that the serum levels of IL-1a in patients with severe GVHD were significantly higher than those in patients with no or mild GVHD (Fig. 5e). Together, these findings indicate that caspase-11 and GSDMD mediates IL-1 $\alpha$ release in GVHD.

Neutralizing IL-1 $\boldsymbol{\alpha}$ attenuates GVHD. Next, we tested whether caspase-11 signaling enhances GVHD in allo-HSCT through releasing IL-1 $\alpha$. Neutralizing IL-1 $\alpha$ using monoclonal antibodies, which almost completely depleted IL-1a in serum (Fig. 6a), led to a marked improvement of GVHD mortality and a significant reduction of GVHD pathological scores (Fig. 6b, c). Meanwhile, administration of IL-1a-neutralizing antibodies significantly decreased serum IFN- $\gamma$ levels (Fig. 6d), and reduced donor T cell expansion in the whole body and small intestine as compared to their WT littermates (Fig. 6e, f). Importantly, neutralizing IL-1 $\alpha$ significantly improved survival in allo-HSCT in WT mice but not in Caspase-11-deficient recipients (Supplementary Fig. 6a). Caspase-11- and GSDMD-induced pyroptosis leads to potassium efflux, resulting in the activation of NLRP3 inflammasome ${ }^{16}$. This event mediates the maturation of IL- $1 \beta$, which has similar biological functions as IL-1 $\alpha$. In agreement with a previous study ${ }^{26}$, Nlrp3 deficiency in recipients delayed but did not reduce mortality when mice were injected with $10^{6}$ allogeneic donor T cells, whereas Caspase-11-deficiency significantly reduced mortality in the same experimental setting (Supplementary Fig. 6b). Caspase11 and GSDMD were also critical for IL-18 release in allo-HSCT (Supplementary Fig. 6c, d). However, genetic deletion of Il-18 failed to affect the survival (Supplementary Fig. 6e). Together, these observations suggest that caspase-11 signaling enhances GVHD, at least in part, through IL-1a.

We next investigated the mechanisms by which IL-1a promotes donor T cell expansion and GVHD severity. Administration of IL-1 $\alpha$-neutralizing antibodies markedly reduced intestinal neutrophil infiltration in allo-HSCT (Supplementary Fig. 7a). In agreement with a previous study ${ }^{1}$, depletion of neutrophils using anti-Ly-6G antibodies significantly improved survival and markedly inhibited donor $\mathrm{T}$ cell expansion in alloHSCT (Supplementary Fig. 7b, c). These findings suggest that IL1a promotes neutrophil infiltration in allo-HSCT, which is critical for donor T cell expansion and GVHD severity.

Caspase-11 deficiency preserves GVL activity. GVL activity is essential for preventing tumor relapse, and is the key to the success of allo-HSCT ${ }^{27}$. Thus, we next determined whether Caspase-11 deficiency in recipients affects GVL maintenance by using alloHSCT model supplemented with EL4 murine T lymphoma cells transduced with luciferase/neo plasmid (EL4-luc) for BLI tumor tracking. Six weeks after MHC-mismatched allo-HSCT, GVHD versus tumor mortality was distinguished by the BLI signal (indicative of tumor load) and weight loss (indicative of GVHD onset). As expected, all recipients of EL4-luc and BM succumbed to tumor within 4 weeks (Fig. 7a). WT recipients of allogeneic T cells all succumbed to GVHD by day 30 after allo-HSCT in parallel with notable weight loss (Fig. 7b). These mice lacked a tumor BLI signal and visible tumor (Fig. 7c). Deletion of Caspase11 in recipients significantly improved both weight maintenance and survival (Fig. 7a-c). Remarkably, none of the Caspase-11deficient mice succumbed to tumor (Fig. 7a and Supplementary Fig. 8). These findings demonstrate that Caspase-11 deficiency does not compromise the GVL effect in allo-HSCT.

\section{Discussion}

Taken together, this study establishes that blocking caspase-11 signaling reduces GVHD while preserving GVL activity. In this scenario, circulating LPS or Gram-negative bacteria activate caspase-11 through GBPs, leading to GSDMD-dependent IL-1 $\alpha$ release. Targeting this pathway by genetic or pharmacological approaches reduced intestinal neutrophil infiltration, tissue damage, and donor T cell expansion in allo-HSCT. Together with 
a

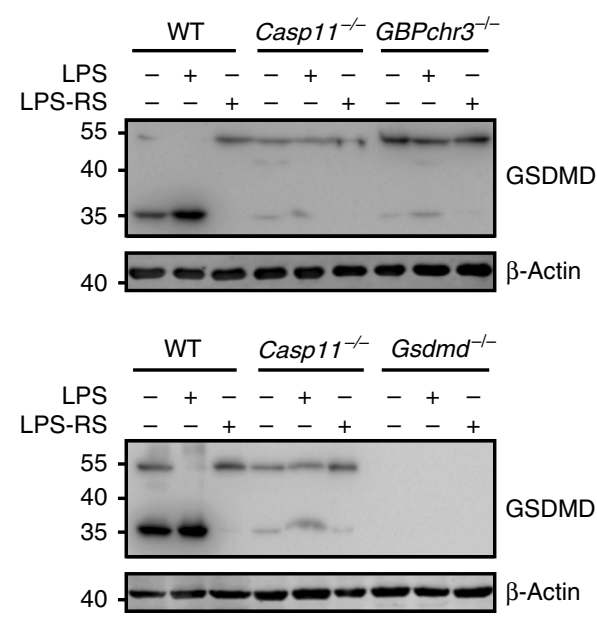

C

$-G s d m d^{+/+}$recipient $(n=5)$
$\quad 0 G s d m d^{-1-}$ recipient $(n=5)$
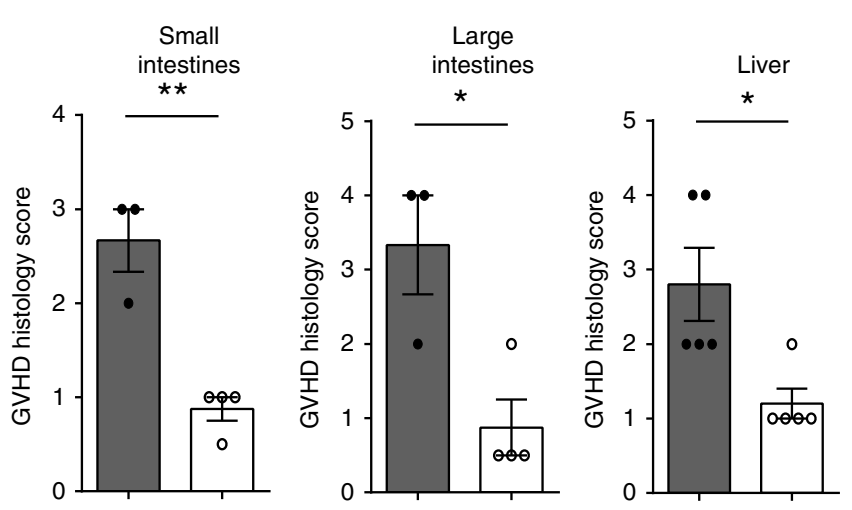

e

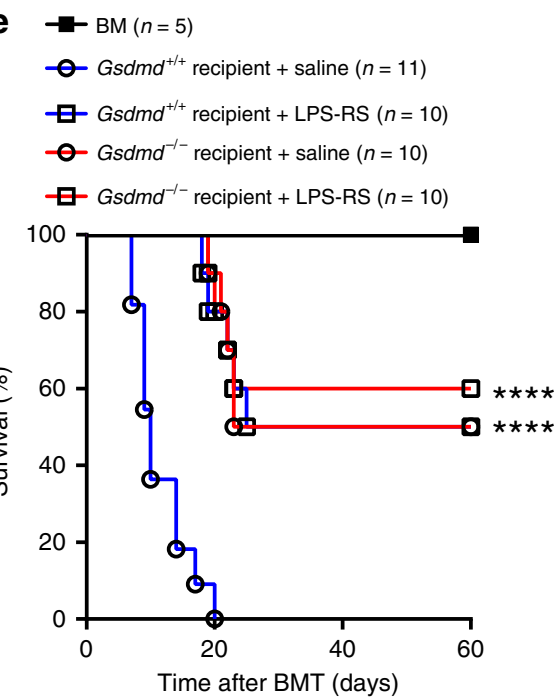

a recent finding that gut microbiota-induced neutrophil recruitment to the intestinal tissue promotes donor $\mathrm{T}$ cell activation and contributes to the pathogenesis of $\mathrm{GVHD}^{1}$, our data shed mechanistic insights into how gut microbiota promotes GVHD in allo-HSCT. The important contribution of gut microbiota to GVHD was first discovered in the 1970s. It then became a common practice to perform intestinal decontamination using orally administered antibiotics in patients undergoing allo$\mathrm{HSCT}^{27}$. Initial reports were promising, but subsequent studies b

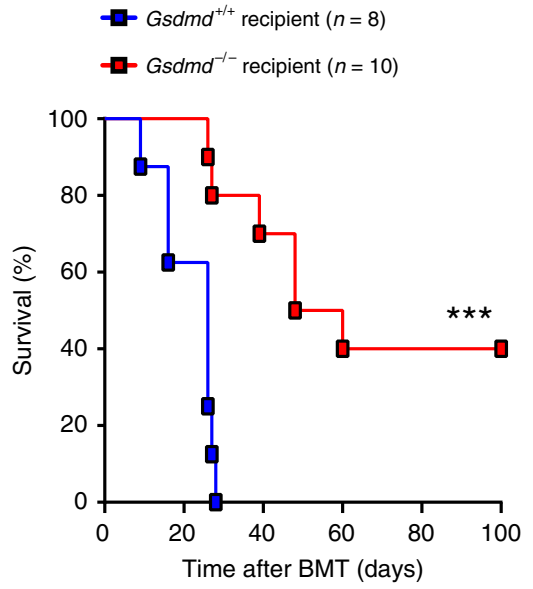

d

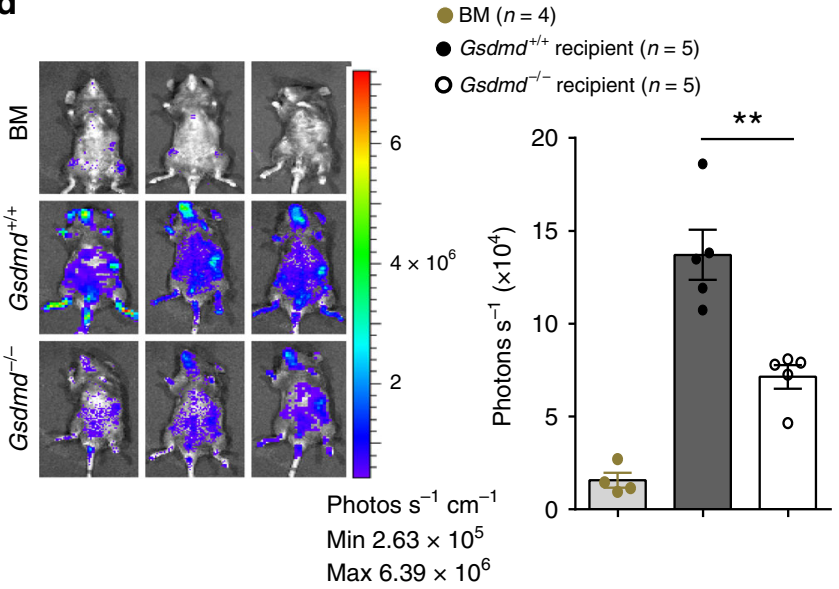

$\mathbf{f}$

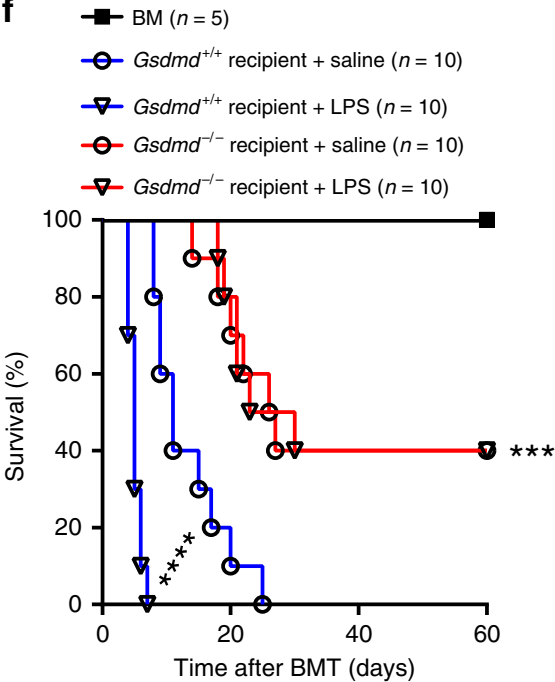

could not confirm a benefit ${ }^{27}$. Broad-spectrum antibiotic use even promotes GVHD by causing dysbiosis ${ }^{28}$. Furthermore, gut microbiota-derived metabolites, such as butyrate, improve intraepithelial cell junctional integrity, decreases apoptosis, and mitigates $\mathrm{GVHD}^{29}$. Therefore, pharmacologically targeting gut microbiota-induced inflammatory responses, such as the caspase11 signaling, might be a more effective option for patients undergoing allo-HSCT than eliminating bacterial colonies in the GI tract through antibiotics. 
Fig. 4 Caspase-11 enhances graft-versus-host disease (GVHD through gasdermin D (GSDMD) in allogeneic hematopoietic stem cell transplantation (alloHSCT). a Immunoblot for GSDMD and $\beta$-actin in the intestine from wild-type (WT) (C57BL/6), Gbpchr3-/- or Gsdmd ${ }^{-/-}$recipients mice with treatment of lipopolysaccharide (LPS) or lipopolysaccharide from the photosynthetic bacterium Rhodobacter sphaeroides (LPS-RS). b Survival of Gsdmd+/+ versus $\mathrm{Gsdmd}^{-/}$- recipient mice (BALB/c $\rightarrow \mathrm{C} 57 \mathrm{BL} / 6$ combination) receiving BALB/c bone marrow and T cells is shown. The experiment was performed twice and the results were pooled. Gsdmd ${ }^{+/+}$versus $\mathrm{Gsdmd}^{-/-}{ }^{\star \star \star} P=0.0002$; differences in animal survival were analyzed by log-rank test. $\mathbf{c}$ Histological analysis of the small intestines, large intestines, and liver from Gsdmd $+/+$ and Gsdmd ${ }^{-/}$- recipient mice receiving BALB/c bone marrow and T cells for GVHD severity on day 14. Data are presented as mean \pm SEM. ${ }^{\star \star} P<0.01 ;{ }^{\star} P<0.05$. An unpaired Student's $t$ test (two-sided) was used. $\mathbf{d}$ Fourteen days post transplant, Gsdmd ${ }^{+/+}$and $\mathrm{Gsdmd}^{-/-}$recipients receiving BALB/c bone marrow and T cells were subjected to intraperitoneal luciferin injection and whole-body bioluminescent imaging (BLI) are depicted. Average representative whole-body images and average bioluminescence intensities \pm SEM were depicted. Data are presented as mean \pm SEM. An unpaired Student's $t$ test (two-sided) was used. ${ }^{\star \star} P<0.01$ (compared with vehicle-treated controls). e Percentage survival of Gsdmd ${ }^{+/+}$and Gsdmd ${ }^{-/-}$recipient mice receiving BALB/c bone marrow and T cells (BALB/c $\rightarrow$ C57BL/6 combination) with treatment of LPS-RS or saline is shown. Differences in animal survival were analyzed by log-rank test. (Gsdmd $d^{+/+}+$saline versus Gsdmd ${ }^{-/-}+$saline,

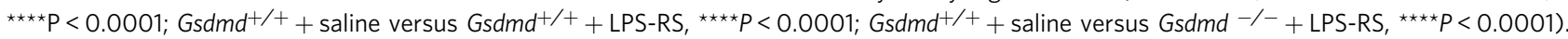

f Percentage survival of Gsdmd $d^{+/+}$and Gsdmd $d^{-/-}$recipient mice receiving BALB/c bone marrow and T cells (BALB/c $\rightarrow$ C57BL/6 combination) with multiple injections of LPS or saline is shown. Differences in animal survival were analyzed by log-rank test. (Gsdmd ${ }^{+/+}+$saline versus Gsdmd ${ }^{-/-}+$saline, ${ }^{\star \star \star} \mathrm{P}=0.0005 ; \mathrm{Gsdm}^{+/+}+$saline versus Gsdmd ${ }^{+/+}+\mathrm{LPS},{ }^{\star \star \star \star} \mathrm{P}<0.0001 ; \mathrm{Gsdmd}^{+/+}+$saline versus Gsdmd $\left.{ }^{-/-}+\mathrm{LPS}{ }^{\star \star \star} P=0.0004\right)$

a
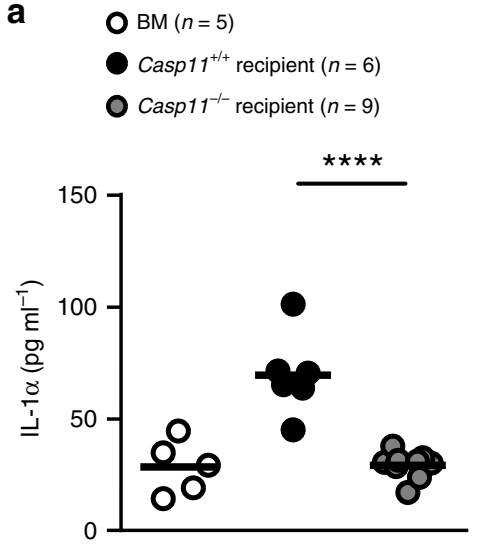

d b
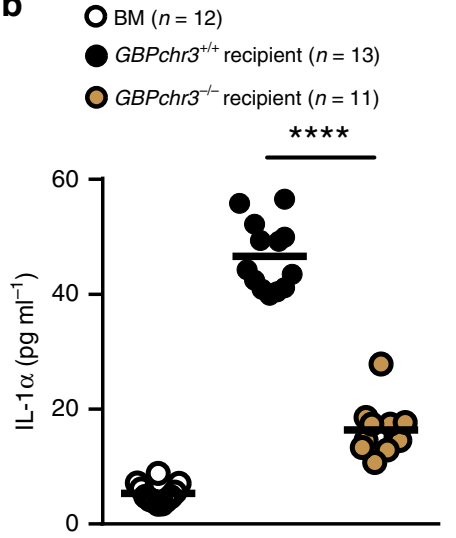

C
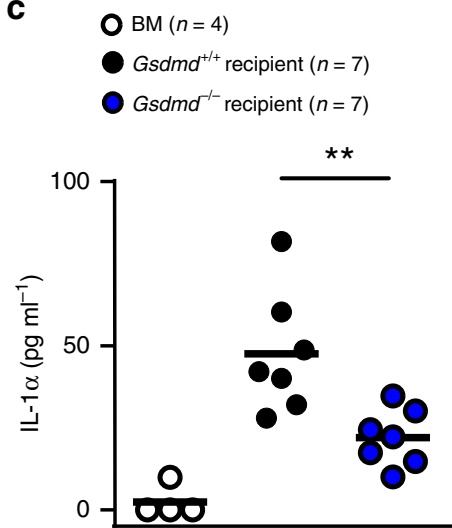

e

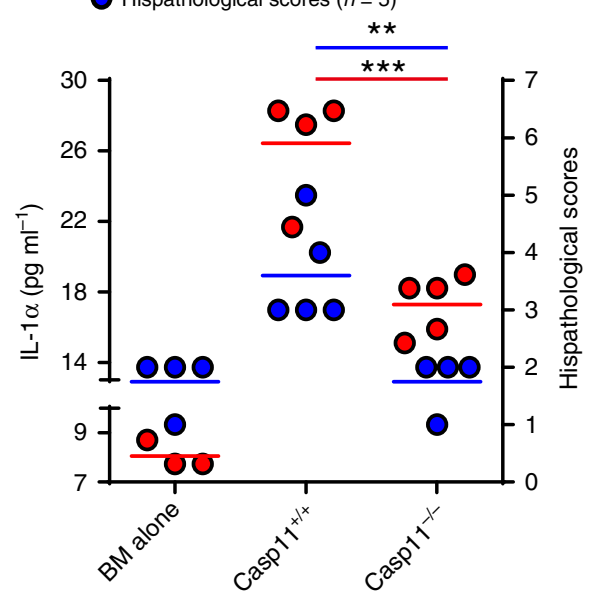

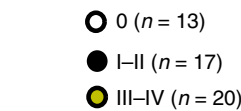

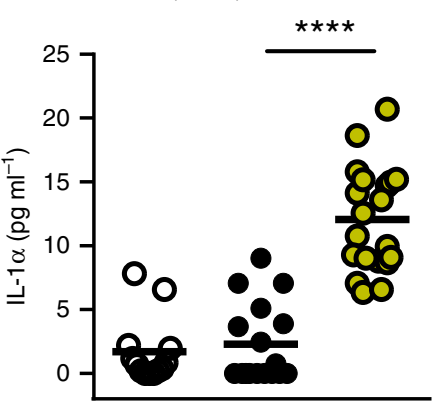

Fig. 5 Caspase-11 and gasdermin D (GSDMD) mediate interleukin-1 $\alpha$ (IL-1 $\alpha$ ) release in graft-versus-host disease (GVHD). a-c Total body irradiation (1100 cGy) was dosed to C57BL/ 6 mice, which were then transplanted with $5 \times 10^{6}$ wild-type (WT) BALB/c T cell-depleted bone marrow (TCD-BM) cells alone or in addition to $5 \times 10^{6}$ total T cells on day 0 . Five days post transplant, serum was subjected to cytokine analysis to quantify serum cytokine concentrations of IL-1 $\alpha$ in Casp11-/- (a) Gbpchr3 ${ }^{-/-}$(b) and Gsdmd ${ }^{-/-}$(c) recipients. An unpaired Student's $t$ test (two-sided) was used. ${ }^{* *} P<0.01$; ${ }^{\star \star \star \star \star} P<0.0001$ (compared with vehicle-treated controls). d The serum IL-1 $\alpha$ levels of Casp11 $1 /+$ and Casp $11^{-/-}$recipients that had undergone allogeneic hematopoietic stem cell transplantation (allo-HSCT) was correlated with GVHD severity. An unpaired Student's $t$ test (two-sided) was used. ${ }^{\star \star} P<0.01$; ${ }^{\star \star \star} P<0.001$. e The levels of IL- $1 \alpha$ in the serum was correlated with the GVHD severity (GVHD grade $0, n=13 ;$ GVHD grade I-II, $n=17 ;$ GVHD grade III-IV, $n=20$ ) in patients after allo-HSCT. ${ }^{\star \star \star \star} P<0.0001$. An unpaired Student's $t$ test (two-sided) was used. The patients' characteristics are detailed in Supplementary Table 1 
a

BM $(n=4)$
Isotype $\lg \mathrm{G}(n=7)$

O IL-1 $\alpha \mathrm{Ab}(n=10)$

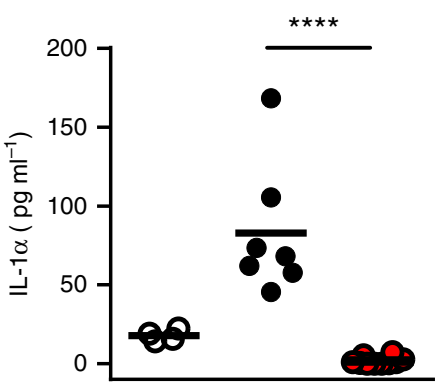

C

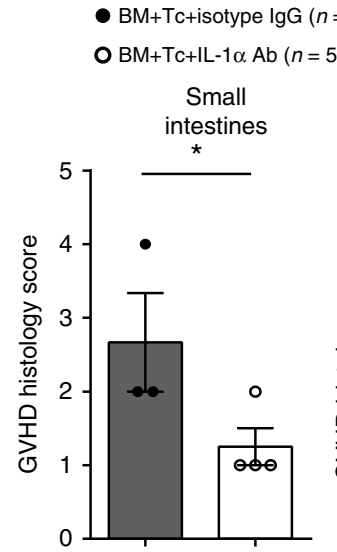

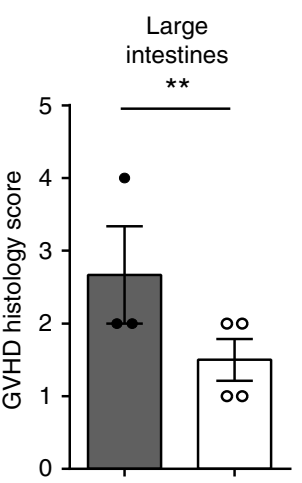

b

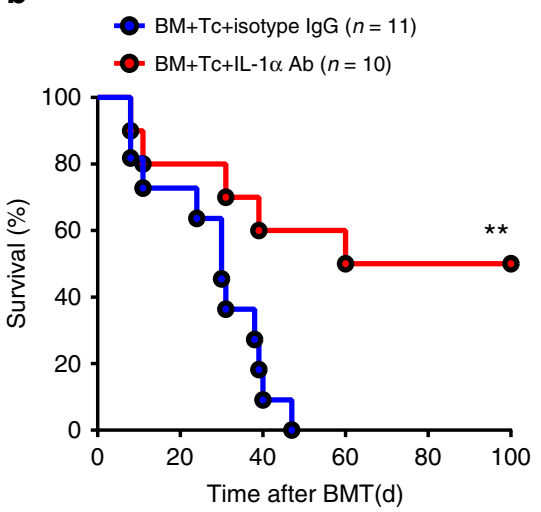

d e

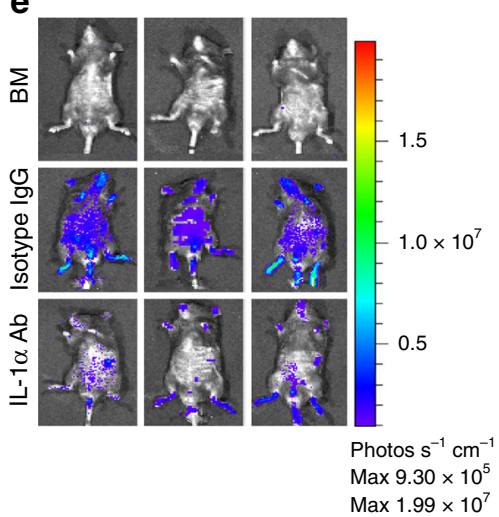

$\operatorname{BM}(n=4)$

- Isotype $\lg G(n=4)$

O IL-1 $\alpha \mathrm{Ab}(n=5)$

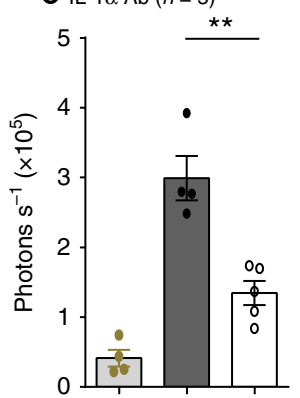

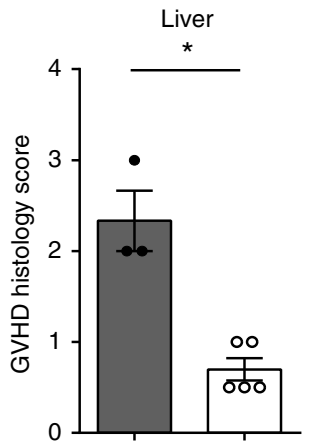

f

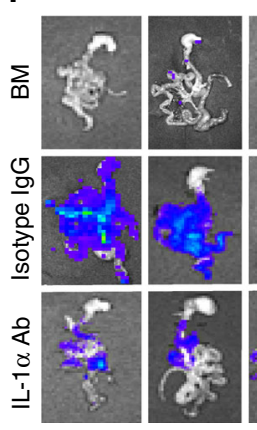

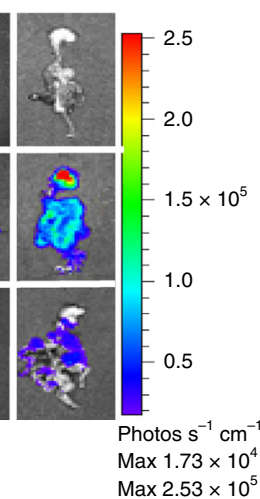

Fig. 6 Neutralizing interleukin-1 $\alpha$ (IL-1 $\alpha$ ) attenuates graft-versus-host disease (GVHD). a Levels of IL-1 $\alpha$ in the serum are shown from mice bone marrow (BM) receiving BALB/c BM or injected with $4 \mu$ g per mouse anti-IL- $1 \alpha$ monoclonal antibody (Ab) or isotype control Ab per mouse on day -1 and day +1 of allogeneic hematopoietic stem cell transplantation (allo-HSCT). Scatter plot represent mean, $n=4(\mathrm{BM}), n=10$ (IL-1 $\alpha$ Ab) and $n=7$ (isotype control) with results pooled from two independent experiments. ${ }^{\star \star \star \star} P<0.0001$, unpaired Student's $t$ test (two-sided). b Survival of C57BL/6 recipients (BALB/c $\rightarrow$ C57BL/6 combination) is shown for a group that received BM/Tc (BM/T cell) with anti-IL-1 $\alpha$ monoclonal Ab or isotype control Ab. BM/Tc + isotype control Ab $(n=11)$ versus BM/Tc + anti-IL-1 $\alpha$ monoclonal $\mathrm{Ab}(n=10),{ }^{\star \star} P=0.0065$. Differences in animal survival were analyzed by log-rank test. c Histological analysis of the small intestines, large intestines, and liver from anti-IL-1 $\alpha$ monoclonal Ab and isotype control Ab mice for GVHD severity on day 14. Data are presented as mean \pm SEM. An unpaired Student's $t$ test (two-sided) was used. ${ }^{\star} P<0.05$; ${ }^{\star \star} P<0.01$. d Levels of interferon- $\gamma$ (IFN- $\gamma$ ) in the serum of the BM/Tc + isotype control Ab $(n=9)$ and BM/Tc + anti-IL-1 $\alpha$ monoclonal Ab recipient mice $(n=9)$ on the day 5 after allo-HSCT. ${ }^{\star \star \star \star} P<$ 0.0001. An unpaired Student's $t$ test (two-sided) was used. e Fourteen days post transplant, BM/Tc + isotype control Ab and BM/Tc + anti-IL-1 $\alpha$ monoclonal $\mathrm{Ab}$ recipients were subjected to intraperitoneal luciferin injection and whole-body bioluminescent imaging (BLI) are depicted. Average representative whole-body images, and average bioluminescence intensities \pm SEM were depicted. An unpaired Student's $t$ test (two-sided) was used. ${ }^{\star \star} P<0.01$ (compared with vehicle-treated controls). f Fourteen days post transplant, BM/Tc + isotype control Ab and BM/Tc + anti-IL-1 $\alpha$ monoclonal Ab recipients were subjected to intraperitoneal luciferin injection, whole-body BLI and BLI of intestines. Average representative ex vivo organ images, and average bioluminescence intensities \pm SEM were depicted. An unpaired Student's $t$ test (two-sided) was used. ${ }^{\star \star \star} P=0.0003$ (compared with vehicletreated controls) 
a

- BM+EL4-Iuc $(n=10)$

$\rightarrow \mathrm{BM}+\mathrm{EL} 4-\mathrm{luc}+\mathrm{Tc}\left(\right.$ Casp $11^{+/+}$recipients $)(n=12)$

$\triangle \mathrm{BM}+\mathrm{EL} 4-\mathrm{luc}+\mathrm{Tc}$ (Casp11 $1^{-/-}$recipients) $(n=11)$

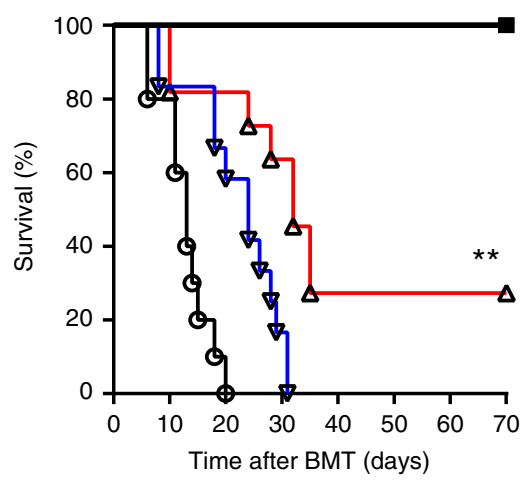

b $\rightarrow$ BM alone $(n=5)$

$\rightarrow$ BM+EL4-luc $(n=10)$

$\rightarrow$ BM+EL4-luc+Tc ( Casp11 $1^{+/+}$recipients) $(n=12)$

$\rightarrow$ BM+EL4-luc+Tc (Casp11 $1^{-/-}$recipients) $(n=11)$

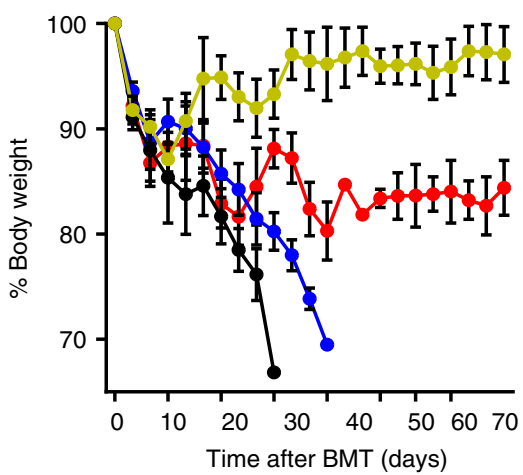

c

BM+EL4-Iuc

Day 14

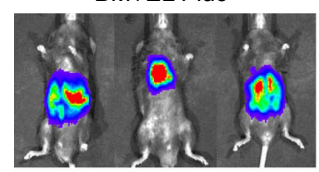

Day 21

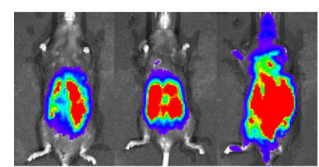

Day 28

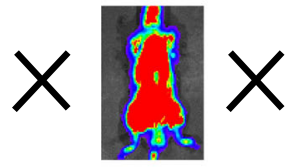

Day 35

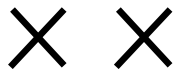

$\times$
BM+Tc+EL4-luc
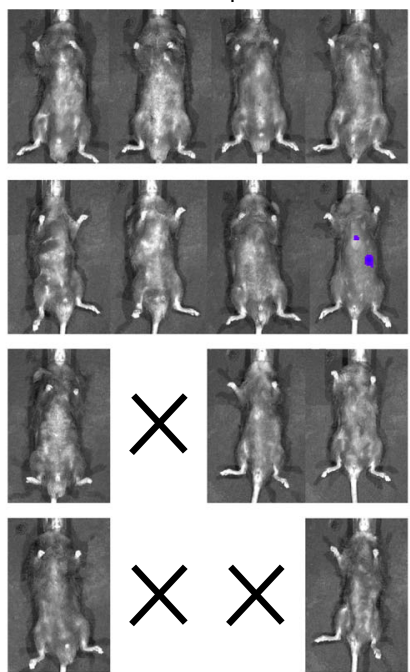

$\times$

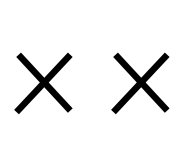

de

$\mathrm{BM}+\mathrm{Tc}+\mathrm{EL} 4-\mathrm{luc}$

Casp $11^{-1-}$ recipients
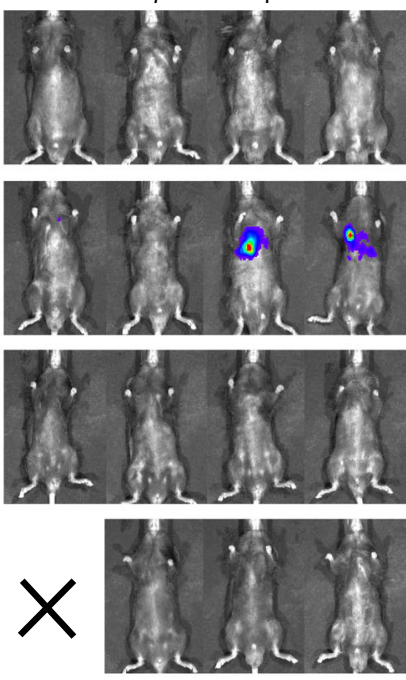

Day 42
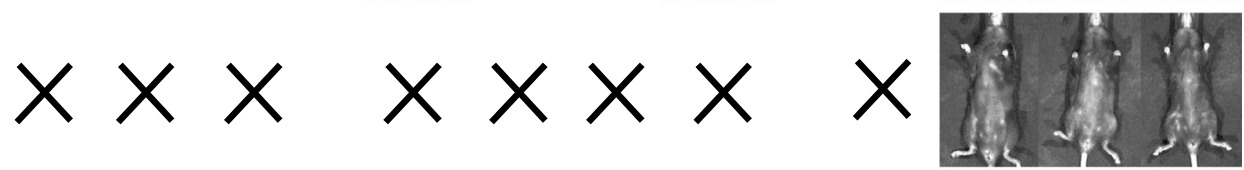

Day 56
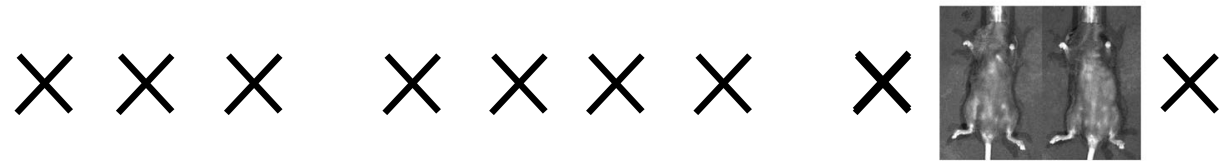

Day $70 \times X$
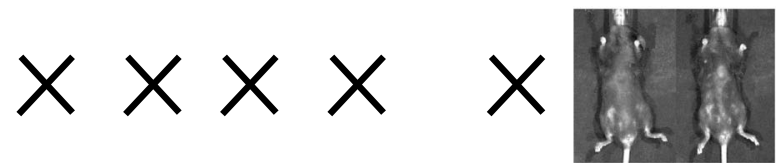

X

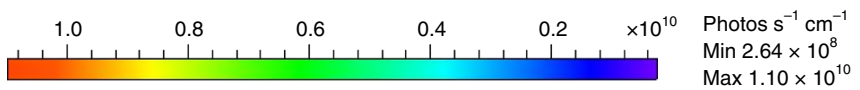

Fig. 7 Caspase-11 deficiency preserves graft-versus-leukemia (GVL) activity. a Survival of Casp11 $1 /+(n=12)$ and Casp11-/- $(n=11)$ recipients had received lethally irradiation ( $1100 \mathrm{cGy}$ ) and transplanted with $5 \times 10^{6} \mathrm{BALB} / \mathrm{c}$ T cell-depleted bone marrow (TCD-BM) cells alone or in addition to $3 \times 10^{6}$ total T cells from BALB/c mice with $3 \times 10^{5}$ EL4 luciferase-transduced lymphoma cells at the time BM transplant (BMT) was shown. Differences in animal survival were analyzed by log-rank test. ${ }^{\star \star} P=0.0039$ (Casp11-/- compared with vehicle-treated controls). b Casp11 ${ }^{+/+}(n=12)$ and Casp11-/- $(n=11)$ recipients were monitored throughout the experimental period for weight change by every 3 to 5 days. c Tumor expansion in Casp11 $1 /+$ and Casp11-/recipients was shown by luciferin intraperitoneal injection and whole-body bioluminescent imaging (BLI) was taken every 14 days 
Type 1 IFN signaling is important for the activation of caspase$11^{11,13,15}$. Priming with poly I:C, a double-stranded RNA mimetic that induces robust type 1 IFN production in a manner similar to that of double-stranded RNA virus, could significantly promote LPS-induced caspase-11 activation and pyroptosis in lung endothelial cells ${ }^{21}$. Further, administration of poly I:C markedly increases caspase-11-dependent lethality in endotoxemia ${ }^{19,20}$. Recent advances reveal a progressive expansion of viral infections over time following allo-HSCT ${ }^{30}$. In particular, the picobirnaviruses, which are double-stranded RNA virus, are strongly associated with severe enteric GVHD ${ }^{30}$. These findings raise an intriguing possibility that allo-HSCT-associated viral infections might enhance GVHD through the caspase-11-GSDMD signaling. It is noteworthy that caspase-1 also mediates GSDMD cleavage. Both gut microbiota and viral infections are able to activate caspase- 1 through canonical inflammasomes. Thus, the roles of caspase-1 in the pathogenesis of GVHD in allo-HSCT merit further investigations.

GVHD remains a major obstacle for the wider usage of alloHSCT $^{1,2}$. To date, most therapeutic or prophylactic strategies to prevent severe GVDH include $\mathrm{T}$ cell depletion, pharmacological inhibition of $\mathrm{T}$ cell activation and proliferation, and intervene with the $\mathrm{T}$ cell-dependent effector phase ${ }^{27}$. These approaches inevitably impair the global host immunity, and thus compromise the GVL activity mediated by donor $\mathrm{T}$ cells, which is the major determinant of the overall outcome after allo-HSCT ${ }^{27}$. In the current study, we show that the absence of caspase-11 signaling reduces GVHD while preserving GVL activity. Therefore, our data have major implications for allo-HSCT, as pharmacological inhibition of caspase-11 signaling might exclusively reduce the unwanted graft-versus-host reactions while sparing the beneficial GVL activity in allo-HSCT.

\section{Methods \\ Human subjects. Serum samples were collected at 30 to 80 days after GVHD} diagnosis, in accordance with research ethics board approval from Xiangya Hos pital of Central South University (CSU). All patients provided informed consent to data collection for research. Patient characteristics are given in Supplementary Table 1. GVHD grading was performed on the basis of Glucksberg grade standard.

Mice. C57BL/6 (H-2Kb, Thy-1.2) and BALB/c (H-2Kd, Thy-1.2) mice were purchased from Hunan SJA Laboratory Animal Co. (Changsha, China). Caspase1 1 $1^{-1-}$ $\mathrm{Il}-18^{-/-}$, and L2G85.BALB/c mice were purchased from Jackson laboratory. The Caspase $11^{-/-}$mice 22 were donated by Timothy R. Billiar (Department of Surgery, University of Pittsburgh Medical Center, Pittsburgh, PA). The GBPchr3 ${ }^{-/-}$mice ${ }^{31}$ were donated by Petr Broz (Focal Area Infection Biology, Biozentrum, University of Basel). The Gsdmd $d^{-1-}$ mice ${ }^{32}$ were donated by Jiahuai Han (State Key Laboratory of Cellular Stress Biology Innovation Center for Cell Signaling Network School of Life Sciences, Xiamen University). The $\mathrm{Nlrp3}^{-1-}$ mice ${ }^{33}$ were donated by Rongbin Zhou (CAS Key Laboratory of Innate Immunity and Chronic Disease, School of Life Sciences, University of Science and Technology of China). All mice except the L2G85.BALB/c mice used in this study are on C57BL/6 background and were housed in specific pathogen-free conditions in the Department of laboratory animals of CSU in China. Mice were used between 6 and 12 weeks of age, and only sex-matched donor-recipient pairs were used. All work was approved and performed according to the Guidelines for Animal Experiments by the Institutional Animal Care and Use Committee of Central South University.

BMT model. BM transplant recipients were given $5 \times 10^{6} \mathrm{BM}$ cells (Biotin antibody CD90.2, eBioscience; MACS negative sorting, STEMCELL, Canada) after lethal irradiation with $11 \mathrm{~Gy}$ (split doses of $2 \times 5.5 \mathrm{~Gy}$ apart to $4 \mathrm{~h}$ ) for the BALB/c $\rightarrow$ C57BL/6 combination and $8 \mathrm{~Gy}$ (split doses of $2 \times 4.0 \mathrm{~Gy}$ apart to $4 \mathrm{~h}$ ) for the $\mathrm{C} 57 \mathrm{BL} / 6 \rightarrow \mathrm{BALB} / \mathrm{c}$ combinations at CSU, China. T cell doses (Biotin antibodies CD11b/TER119/B220/CD49b/CD24/CD19b, eBioscience; MACS negative enrichment, STEMCELL, Canada) varied depending on the transplant model C57Bl/6 $\geq$ $\mathrm{BALB} / \mathrm{c}\left(8 \times 10^{5}\right)$ and $\mathrm{BALB} / \mathrm{c} \geq \mathrm{C} 57 \mathrm{Bl} / 6\left(2-5 \times 10^{6}\right)$.

Histopathology scoring of acute GVHD. Slides of small bowel and large bowel samples collected on day 14 were stained with hematoxylin/eosin and scored by experienced pathologists blinded to the treatment groups. Our GVHD grading system was as follows: liver-grade 0.5 , focal portal lymphoid infiltrate; grade 1 , widespread portal lymphoid infiltrate; grade 2, focal bile duct invasion or cellular injury; grade 3, multiple foci of bile duct injury and regeneration; grade 4 , widespread injury and destruction of bile ducts. Small and large intestines-grade 0.5 , occasional or rare necrotic cells in glands or crypts; grade 1, multiple foci of necrotic cells in glands or crypts; grade 2, necrosis involving several crypts or glands with focal abscess formation in crypts; grade 3, widespread crypt abscesses with focal glandular destruction; grade 4 , loss of mucosa with granulation tissue response.

T cell lymphoma model. To investigate GVL activity of transferred donor T cells, we used luciferase transgenic EL4 (EL4-luc) T cell leukemia that had been previously demonstrated to migrate primarily to the $\mathrm{BM}$, with secondary infiltration of the spleen and other lymphoid organs when injected intravenously at the time of BMT. BALB/ $\mathrm{c} \rightarrow \mathrm{B} 6$ recipients received $5 \times 10^{3}$ luciferase/neo plasmid-transduced EL4 T cell lymphoma cells (EL4-luc) at the time of BMT. Tumor mortality and GVHD mortality were distinguished by BLI for tumor load and weight loss indicative of GVHD. BLI data were analyzed and quantified using the Living Image Software (Xenogen).

Chemotherapy treatment. Mice received BU $\left(10 \mathrm{mg} \mathrm{kg}^{-1} \mathrm{day}^{-1}\right.$ from days -7 to -4 ; Busilvex, Pierre Fabre Pharma, Germany) and CY $\left(100 \mathrm{mg} \mathrm{kg}^{-1} \mathrm{day}^{-1}\right.$ from days -3 to -2 ; Endoxan, Baxter Healthcare, USA) i.p. (BU/CY). Day of stem cell injection was assigned as day 0 and the days before stem cell injection are numbered backwards.

Drug treatment. The mice were injected i.p. with $4 \mu \mathrm{g}$ per mouse a monoclonal anti-mouse IL-1a antibody (Ab) (BioLegend, Germany) or isotype control Ab (rat IgG2a, BioLegend, Germany) in $100 \mu \mathrm{l}$ of NS $24 \mathrm{~h}$ before TBI (total body irradiation) and $24 \mathrm{~h}$ after BMT.

BLI imaging. For BLI studies, mice were injected i.p. with luciferin $\left(10 \mu \mathrm{g} \mathrm{g}^{-1}\right.$ body weight). Ten minutes later mice were imaged using an IVIS100 charge-coupled device (CCD) imaging system (Xenogen, Alameda, CA) for 5 min. Expansion was quantified in photons $\mathrm{s}^{-1} \mathrm{~cm}^{-2}$. Imaging data were analyzed and quantified with Living Image Software (Xenogen)

Conventional histology and immunohistochemistry. Paraffin-embedded sections of $5-\mu \mathrm{m}$ thickness were mounted on microscope slides. After fixing, the primary $\mathrm{Ab}$ (anti-MPO Abs, Abcam, USA) was applied. Sections were then incubated with a specific secondary biotinylated Ab. Streptavidin horseradish peroxidase and 3,3'-diaminobenzidine (Abcam, USA) were used according to the manufacturer's instructions, and sections were counterstained with hematoxylin (Thermo Scientific, USA).

Cytokine measurements. The levels of IL-1 $\alpha$ and IFN- $\gamma$ were analyzed from the serum with the eBioscience Inflammation Kit (eBioscience, USA) and used according to the manufacturer's instructions.

Isolation of lamia propria lymphocytes. Mice were sacrificed and intestines were flushed with $\mathrm{PBS}$ Ca-/Mg- after dissection of fat and mesenteric tissue and excision of Peyer's patches. Pieces $(0.5 \mathrm{~cm})$ of longitudinally opened and $\mathrm{PBS} \mathrm{Ca}-/ \mathrm{Mg}_{\text {-washed }}$ intestine were incubated with Hank's balanced salt solution (HBSS) containing $2 \mathrm{mM}$ EDTA, $10 \mathrm{mM}$ HEPES, 5\% fetal bovine serum (FBS), and $1 \mathrm{mM}$ dithiothreitol. After 15 min of incubation on a shaker at $37^{\circ} \mathrm{C}$, intraepithelial lymphocytes and epithelium were separated via $100-\mu \mathrm{m}$ filters. Tissues were digested in the $\mathrm{HBSS}^{\mathrm{Ca}+/ \mathrm{Mg}+}$ medium (Thermo Fisher Scientific) containing $10 \mathrm{mM} \mathrm{HEPES}$, and $5 \% \mathrm{FBS}$ at $37^{\circ} \mathrm{C}$ in a $5 \%$ $\mathrm{CO}_{2}$ incubator for $0.5 \mathrm{~h}$. Then, intestinal lamia propria lymphocytes (LPLs) were isolated using the gentleMACS Program m_intestine (gentleMACS Dissociator \#130 093-235) and Intestinal LPL Isolation Kit (Lot: 130-097-410, Miltenyi, Germany).

Flow cytometry. Staining was performed in the presence of purified Ab to CD16/ 32 at saturation to block nonspecific staining. For flow analysis, cells were lysed and fixed with $1 \times$ PhosphoFlow Lyse/Fix Buffer for $15 \mathrm{~min}$ at $37^{\circ} \mathrm{C}$, and then permeabilized with PhosFlow Perm Buffer III on ice for $1 \mathrm{~h}$ (all reagents were from eBioscience USA).

Proximity-ligation assay. PLA Kit (Sigma, USA) was used to study the interaction between LPS and caspase-11 in mouse intestinal tissue. This unique method is able to visualize molecular interactions in situ. After fixation with $4 \%$ formaldehyde and permeabilization with $0.1 \%$ Triton for $10 \mathrm{~min}$, cells were then incubated overnight with primary antibodies against LPS (mouse monoclonal 2D7/1) or caspase-11 (rat monoclonal 17D9). Briefly, after incubation with primary antibodies, cells were incubated with a combination of corresponding PLA probes, and secondary antibodies were conjugated to oligonucleotides (mouse PLUS and rat MINUS for LPS and caspase-11 interaction). Subsequently, ligase was added forming circular DNA strands when PLA probes were bound in close proximity, along with polymerase and oligonucleotides to allow rolling circle amplification. Fluorescent dyelabeled probes complementary in sequence to the rolling circle amplification product were hybridized to the rolling circle amplification product. Thus, each individual pair of proteins generated a spot that could be visualized using fluorescent microscopy. Images were taken using a Leica confocal laser scanning microscope and analyzed using the ImageJ software. In some experiments, cells were incubated with antibodies against mouse pan-cytokeratin (Clone: AE1/AE3, Lot: 2086277; Thermo, USA), the epithelial marker, after PLA procedure. 
Immunoblot. Proteins were extracted from intestinal tissues. Protein samples were separated by $12 \%$ sodium dodecyl sulfate-polyacrylamide gel electrophoresis and transferred onto PVDF membranes (Millipore). Antibodies against mouse GSDMD (Abcam, USA) was used at 1:1000. Blots were normalized to $\beta$-actin (CST, USA) expression.

Statistical analyses. All data were analyzed using the GraphPad Prism software (version 6.01). Data were analyzed using Student's $t$ test for comparison between two groups. Survival data were analyzed using the log-rank test. A $p$ value $<0.05$ was considered statistically significant for all experiments. All values are presented as the mean \pm SEM (error bars).

\section{Data availability}

The authors declare that all data supporting the findings of this study are available within the article and its Supplementary Information Files or from the corresponding author upon reasonable request.

Received: 16 September 2018 Accepted: 8 August 2019

Published online: 06 September 2019

\section{References}

1. Schwab, L. et al. Neutrophil granulocytes recruited upon translocation of intestinal bacteria enhance graft-versus-host disease via tissue damage. Nat. Med. 20, 648-654 (2014).

2. Koyama, M. et al. Recipient nonhematopoietic antigen-presenting cells are sufficient to induce lethal acute graft-versus-host disease. Nat. Med. 18 , 135-142 (2011).

3. Hill, G. R. et al. Total body irradiation and acute graft-versus-host disease: the role of gastrointestinal damage and inflammatory cytokines. Blood $\mathbf{9 0}$, 3204-3213 (1997).

4. Price, K. S. et al. Progressive accumulation of bacterial lipopolysaccharide in vivo during murine acute graft-versus-host disease. Scand. J. Immunol. 45, 294-300 (1997).

5. Cooke, K. R. et al. LPS antagonism reduces graft-versus-host disease and preserves graft-versus-leukemia activity after experimental bone marrow transplantation. J. Clin. Invest. 107, 1581-1589 (2001).

6. Cohen, J. et al. Antibody titres to a rough-mutant strain of Escherichia coli in patients undergoing allogeneic bone-marrow transplantation. Evidence of a protective effect against graft-versus-host disease. Lancet (Lond., Engl.) 1, 8-11 (1987).

7. Bayston, K. et al. Anti-endotoxin antibody for prevention of acute GVHD. Bone Marrow Transplant. 8, 426-427 (1991).

8. Imado, T. et al. The protective role of host Toll-like receptor-4 in acute graftversus-host disease. Transplantation 90, 1063-1070 (2010).

9. Shi, J. et al. Inflammatory caspases are innate immune receptors for intracellular LPS. Nature 514, 187-192 (2014).

10. Kayagaki, N. et al. Non-canonical inflammasome activation targets caspase11. Nature 479, 117-121 (2011).

11. Rathinam, V. A. et al. TRIF licenses caspase-11-dependent NLRP3 inflammasome activation by Gram-negative bacteria. Cell 150, 606-619 (2012).

12. Aachoui, Y. et al. Caspase-11 protects against bacteria that escape the vacuole. Science (New York, NY) 339, 975-978 (2013).

13. Meunier, E. et al. Caspase-11 activation requires lysis of pathogen-containing vacuoles by IFN-induced GTPases. Nature 509, 366-370 (2014).

14. Vanaja, S. K. et al. Bacterial outer membrane vesicles mediate cytosolic localization of LPS and caspase-11 activation. Cell 165, 1106-1119 (2016).

15. Man, S. M. et al. IRGB10 liberates bacterial ligands for sensing by the AIM2 and cspase-11-NLRP3 inflammasomes. Cell 167, 382-396.e317 (2016).

16. Kayagaki, N. et al. Caspase-11 cleaves gasdermin D for non-canonical inflammasome signalling. Nature 526, 666-671 (2015).

17. Shi, J. et al. Cleavage of GSDMD by inflammatory caspases determines pyroptotic cell death. Nature 526, 660-665 (2015).

18. Ding, J. et al. Pore-forming activity and structural autoinhibition of the gasdermin family. Nature 535, 111-116 (2016)

19. Hagar, J. A. et al. Cytoplasmic LPS activates caspase-11: implications in TLR4independent endotoxic shock. Science (New York, NY) 341, 1250-1253 (2013).

20. Kayagaki, N. et al. Noncanonical inflammasome activation by intracellular LPS independent of TLR4. Science (New York, NY) 341, 1246-1249 (2013).

21. Cheng, K. T. et al. Caspase-11-mediated endothelial pyroptosis underlies endotoxemia-induced lung injury. J. Clin. Invest. 127, 4124-4135 (2017).

22. Deng, M. et al. The endotoxin delivery protein HMGB1 mediates caspase-11dependent lethality in sepsis. Immunity 49, 740-753.e747 (2018).

23. Kerur, N. et al. cGAS drives noncanonical-inflammasome activation in agerelated macular degeneration. Nat. Med. 24, 50-61 (2018).
24. Demon, D. et al. Caspase-11 is expressed in the colonic mucosa and protects against dextran sodium sulfate-induced colitis. Mucosal Immunol. 7, 1480-1491 (2014)

25. Santos, J. C. et al. LPS targets host guanylate-binding proteins to the bacterial outer membrane for non-canonical inflammasome activation. EMBO J. 37 https://doi.org/10.15252/embj.201798089 (2018).

26. Jankovic, D. et al. The Nlrp3 inflammasome regulates acute graft-versus-host disease. J. Exp. Med. 210, 1899-1910 (2013).

27. Penack, O. et al. Graft-versus-host disease: regulation by microbeassociated molecules and innate immune receptors. Blood 115, 1865-1872 (2010).

28. Shono, Y. et al. Increased GVHD-related mortality with broad-spectrum antibiotic use after allogeneic hematopoietic stem cell transplantation in human patients and mice. Sci. Transl. Med. 8, 339ra371 (2016).

29. Mathewson, N. D. et al. Gut microbiome-derived metabolites modulate intestinal epithelial cell damage and mitigate graft-versus-host disease. Nat. Immunol. 17, 505-513 (2016).

30. Legoff, J. et al. The eukaryotic gut virome in hematopoietic stem cell transplantation: new clues in enteric graft-versus-host disease. Nat. Med. 23 1080-1085 (2017)

31. Yamamoto, M. et al. A cluster of interferon-gamma-inducible p65 GTPases plays a critical role in host defense against Toxoplasma gondii. Immunity 37 , 302-313 (2012).

32. He, W. T. et al. Gasdermin D is an executor of pyroptosis and required for interleukin-1beta secretion. Cell Res. 25, 1285-1298 (2015).

33. Mariathasan, S. et al. Differential activation of the inflammasome by caspase-1 adaptors ASC and Ipaf. Nature 8, 213-218 (2004).

\section{Acknowledgements}

This study was supported by the National Natural Science Foundation of China (No. 81930059 (B.L.), No. 81470345 (B.L.) and No. 81971893(Y.T.)), National Key Scientific Project 2015CB910700 (B.L.), and Innovation-Driven Scientific Project of CSU (B.L.). We are grateful to Dr. Timothy R. Billiar, Dr. Petr Broz, Dr. Jiahuai Han, Dr. Rongbin Zhou for providing us the Caspase11 $1^{-/-}, G B P c h r 3^{-/-}, G s d m d^{-/-}$, and Nlrp $3^{-/-}$mice. We thank Q. Xue for managing mouse colonies and research assistance. We also thank Institute of Medical Science of Xiangya Hospital, Central South University for technical assistance on flow cytometry.

\section{Author contributions}

B.L. conceived the project, designed the experiments, supervised the study, and wrote the paper; Y.L. designed and performed the experiments; R.M., X.W., S.Y., Q.X. D.S., and M.D. performed the experiments; Y.L., Y.X., Y.T. and J.W. analyzed and interpreted the data; Y.L., R.M., and X.W. made the figures; and X.X., F.C., H.W., Q.W. and T.R.B. assisted in data interpretation and edited the manuscript.

\section{Additional information}

Supplementary Information accompanies this paper at https://doi.org/10.1038/s41467019-11895-2.

Competing interests: The authors declare no competing interests.

Reprints and permission information is available online at http://npg.nature.com/ reprintsandpermissions/

Peer review information: Nature Communication would like to thank James Ferrara, Emad Alnemri, and other anonymous reviewer(s) for their contributions to the peer review of this work.

Publisher's note: Springer Nature remains neutral with regard to jurisdictional claims in published maps and institutional affiliations.

cc (i) Open Access This article is licensed under a Creative Common Attribution 4.0 International License, which permits use, sharing, adaptation, distribution and reproduction in any medium or format, as long as you give appropriate credit to the original author(s) and the source, provide a link to the Creative Commons license, and indicate if changes were made. The images or other third party material in this article are included in the article's Creative Commons license, unless indicated otherwise in a credit line to the material. If material is not included in the article's Creative Commons license and your intended use is not permitted by statutory regulation or exceeds the permitted use, you will need to obtain permission directly from the copyright holder. To view a copy of this license, visit http://creativecommons.org/ licenses/by/4.0/.

(c) The Author(s) 2019 